Review

\title{
Towards Theranostic Multicompartment Microcap- sules: in-situ Diagnostics and Laser-induced Treatment
}

\author{
Ranhua Xiong ${ }^{1}$, Stefaan J. Soenen ${ }^{1}$, Kevin Braeckmans ${ }^{1,2}$, Andre G. Skirtach ${ }^{2,3,4 \bowtie}$ \\ 1. Lab. of General Biochemistry and Physical Pharmacy, Ghent University, Ghent 9000, Belgium; \\ 2. Centre for Nano-Bio (NB)-photonics, Ghent University, Ghent 9000, Belgium; \\ 3. Department of Molecular Biotechnology, Ghent University, Ghent 9000, Belgium; \\ 4. Max-Planck Institute of Colloids and Interfaces, Potsdam 14424 Germany.
}

$\bowtie$ Corresponding author: Andre.Skirtach@UGent.be.

(C) Ivyspring International Publisher. This is an open-access article distributed under the terms of the Creative Commons License (http://creativecommons.org/ licenses/by-nc-nd/3.0/). Reproduction is permitted for personal, noncommercial use, provided that the article is in whole, unmodified, and properly cited.

Received: 2013.01.09; Accepted: 2013.02.05; Published: 2013.02.19

\begin{abstract}
Paving the way towards the application of polyelectrolyte multilayer capsules in theranostics, we describe diagnostic multi-functionality and drug delivery using multicompartment polymeric capsules which represent the next generation of drug delivery carriers. Their versatility is particularly important for potential applications in the area of theranostics wherein the carriers are endowed with the functionality for both diagnostics and therapy. Responsiveness towards external stimuli is attractive for providing controlled and on-demand release of encapsulated materials. An overview of external stimuli is presented with an emphasis on light as a physical stimulus which has been widely used for activation of microcapsules and release of their contents. In this article we also describe existing and new approaches to build multicompartment microcapsules as well as means available to achieve controlled and triggered release from their subcompartments, with a focus on applications in theranostics. Outlook for future directions in the area are highlighted.
\end{abstract}

Key words: theranostics, multicompartment, capsules, stimuli, laser, nanoplasmonics.

\section{Introduction}

Theranostics is an emerging area which combines multifunctionalization [1] for diagnostics and therapy, while nano and micromaterials are developed to enable both functionalities. Such a synergy, particularly if the materials allow to perform diagnosis and therapy simultaneously, will allow physicians not only to detect diseases, but ideally will also permit them to administer proper medicines at the appropriate time, location and dose in the body and to fight many diseases including cancer [2]. Laser light is particularly interesting for theranostics [3] largely due to its potential in phototherapy [4]. Application of light to theranostics opens extensive opportunities in pharmaceutical research to monitor effects of drugs, their distribution, release, and dose thus representing the next step [5] for nanomedicine [6].
A very interesting class of materials for theranostics is multicompartment polyelectrolyte multilayer capsules, which are responding to different stimuli [7]. Multicompartmentalization is an attractive strategy because it allows for assembling or encapsulating several types of molecules simultaneously in the same carrier. Such an approach is particularly suitable for theranostics as some compartments are intended for diagnostics, while other subcompartments are to be used for therapy. A generalization of various approaches to multicompartmentalization has been recently presented by Delcea et al [1]. The structure of multicompartment [8] delivery vehicles can be very diverse $[9,10]$ including a hybrid microcapsule-liposome system with liposomes [11-13] or self-assembled construction [14] including those 
based on polymeric systems [15]. Another desirable feature of a theranostic carrier is the induction of on-demand drug release $[16,17]$ to initiate a therapeutic treatment. Various stimuli are available for release [18] and these stimuli can be logically divided into physical, chemical and biological ones.

Endogenous biological stimuli are most often used for permeability [19] control as well as for performing a relatively slow release [20],[21] of pharmaceutical cargo based on enzymatic degradation [22, 23]. One example is enzyme-catalyzed carrier degradation, which can also be accomplished using sophisticated coupled mechanisms [24]. Another class of release mechanisms, at least for in-vitro experiments, is based on chemical reactions, i.e. chemical stimuli. A number of chemical stimuli such as differences in salt concentration, solvent types or $\mathrm{pH}$ levels [25] can be used for inducing cargo release. While chemical stimuli can be quite effective and quick, they are typically not suited for in-vivo applications. In this regard, physical stimuli such as light, magnetic fields and mechanical forces are more promising for application in a biological context since they have little or no effect on the surrounding medium and can be well-controlled due to possibilities to adjust the stimulating fields' strength. Laser light has a special niche in this area as an external physical stimulus since it can be very well controlled spatially and temporally so that high resolution imaging is possible for diagnostics with highly localized controlled activation of the therapeutic action. In addition, also from a research perspective it is of interest since it can be easily combined with existing optical microscopes, thus allowing detailed investigation of light-carrier interaction in a biological environment.

In what follows we will describe approaches for theranostics in which multicompartmentalization of microcapsules can be logically applied for diagnostics and treatment.

\section{Diagnostics and Treatment}

Diagnostics using materials encapsulated in some compartments and therapy performed by drugs encapsulated in other compartments is the corner-stone of theranostic applications. In order to conduct the diagnostics part effectively, it is essential to develop and apply appropriate imaging. Here one would clearly distinguish two types of imaging modalities: a) those used in cell culture work, and b) those applicable in-vivo. Microscope-based approaches prove to be the most effective ones for cell cultures. Here contrast agents are incorporated into some compartments of the carrier according to the intended imaging modality to be used. Cell labeling is achieved by fluorophores, which permit visualization of cytoskeleton, nucleus, and other subcompartments as well as visualization of individual molecules with super-resolution. An interesting application is label-free, Raman imaging [26]. A number of other approaches can be used for in-vivo imaging; these include: X-ray, ultrasound, computer tomography (x-ray based), positron emission tomography, magnetic resonance imaging, and their combination often referred to as multimodal imaging.

Diagnosing various parameters can be intended for prevention or treatment, the latter being an inherent part of theranostics. Many different parameters can be diagnosed including acidity $(\mathrm{pH})$ and ionic contents [27], glucose and other biomarkers, products of enzyme-catalyzed reactions, host-pathogen and other biomolecular interactions, bacteria, etc. Some of these parameters, like $\mathrm{pH}$, can provide very important information on functions of cells, organs, and eventually the body. And these parameters can be monitored by molecules encapsulated into different subcompartments of multicompartmental capsules. Also, with regard to therapeutic applications, some of the above methods can be used not only for direct visualization, but also for activation and/or release of active bio-molecules from diagnostic subcompartments of multicompartmental capsules. The treatment modality is administered based upon results obtained from the diagnostic assays.

The following sections describe various approaches describing different types of multicompartmental microcapsules and methods of remote control [28] for on-site specific release.

\section{Multicompartment microcapsules}

Multicompartment microcapsules have been designed almost specifically with theranostics in mind. Diversity of materials for encapsulation into different compartments and variety of multicompartment morphologies and structures make multicompartmentalization a very versatile approach. Various different approaches can be used for assembling multicompartment capsules, namely: a) assembly from previously pre-formed different subcompartments; $b$ ) direct synthesis of capsules (or templates on which capsules are assembled); c) a combination of synthesis and assembly from previously pre-formed subcompartments [1].

Here we will discuss four of the most important multicompartmentalization morphologies, as illustrated in Figure 1. First, concentric structures can be obtained in which multiple compartments are built (concentrically) around the first compartment, see Figure 1 (a) [1]. The first compartment can be built around a particle or a capsule, while subsequent concentric subcompartments are further built around this 
first block. Synthetic routes (for example, calcium carbonate) are used in this case for fabrication. This process can be separated in several steps. First the inner core is synthesized, which can be used as a precursor for outer concentric shells that are synthesized consequently. During further synthesis of concentric subcompartments around the primary subcompartment (or the inner core), some single compartment cores can also appear [29]: then functionalization of concentric multicompartment microcapsules by magnetic nanoparticles can be used for separation purposes.

The second type of multicompartment capsules is the so-called pericentric type, Figure 1 (b) [1]. In this approach, multiple compartments are adsorbed or connected (possibly chemically) to the first and main carrier capsule. This is a versatile approach amenable for mass production since it is based on a relatively simple assembly of pre-fabricated particles/capsules. The interaction of subcompartments takes place due to electrostatic forces, steric entrapment or hydrogen bonding. To this end, subcompartments are typically modified by oppositely charged polymers or molecules capable of inducing the above interactions.

The third type of multicompartment capsules are innercentric, in which case various compartments are incorporated into the interior of the first and main compartment, see Figure 1 (c) [1]. The subcompartments can be synthesized during the assembly process of this type of multicompartment capsules. Although the feasibility was already demonstrated, the relative complexity of this approach is a potential obstacle for widespread use.

In the fourth and last approach acentric structures are prepared and described [1, 14]. Here, the subcompartments can be either synthesized during the assembly process of the multicompartment capsules, or they might be already pre-formed. Many different implementations of such an approach are possible [30]. Its key attribute is non-uniformity or non-symmetry of the structure. Figure 1 (d) shows several structures wherein two different subcompartments are connected, thus forming an elongated two-compartment capsule.

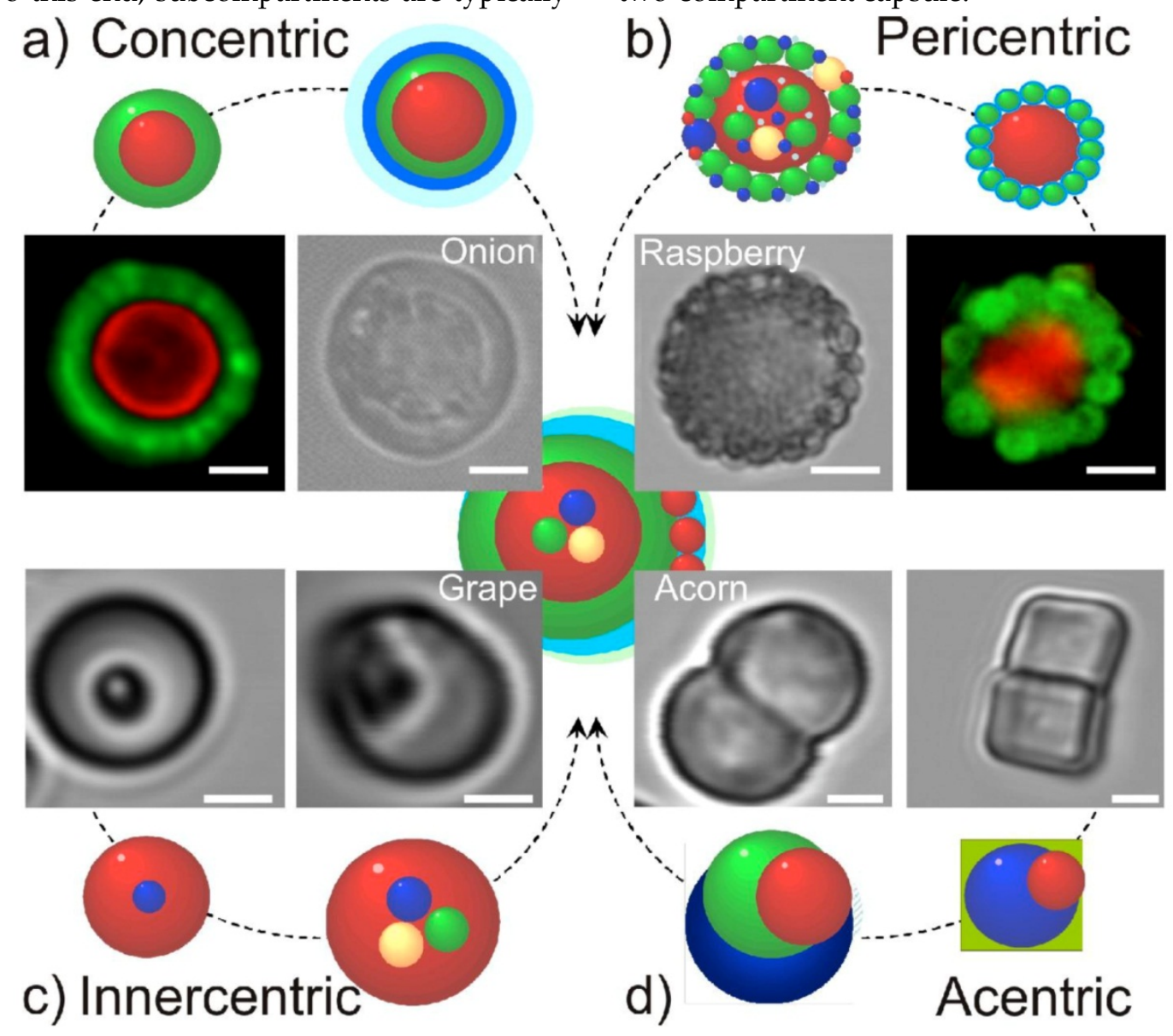

Figure I. Overview and the road-map for future directions of multicompartment microcapsules.Four different approaches are identified in the schematics: a) concentric, b) pericentric, c) innercentric, and d) acentric. The structure in the middle incorporates all four approaches. The corresponding confocal microscope images of the first steps in each direction are also presented. Scale bars correspond to $2 \mathrm{~mm}$. [I] Reproduced by permission of Wiley-VHC. 


\section{Anisotropic multicompartment micro- capsules}

Anisotropy of drug delivery vehicles, as in the case for the acentric capsules discussed above, is an important characteristic for theranostics. Anisotropy of delivery carriers is a very desirable feature for pulmonary drug delivery [31] because elongated carriers possess the same aerodynamic diameter as an equivalent spherical carrier but allows for delivery of a larger amount of cargo. Porosity of carriers is another desirable feature that is of importance for optimizing the aerodynamic diameter as it allows tuning the effective density. In this regard, calcium carbonate particles or hydrogels[32] are attractive candidates.

Early attention to anisotropic particles was given by De Gennes providing a significant impetus for development. Particles in which one half is distinct from the other are called Janus particles [33]. Chemical synthesis and modification are two of the most common routes of controlling [34] the patchiness [35] of Janus particles. Using these routes, various aniso- tropic structures and approaches were reported using microfluidics [36], electroless deposition [37], microcontact printing [38], grafting [39], and plasma polymerization [40].

Anisotropic multicompartment structures have been recently reported using a method of partially shielding a lower part of particles/capsules incorporated into soft films [30]. In such an approach, particles or capsules are sedimented onto the films leaving the upper part non-protected. In the subsequent step, (typically) smaller particles/capsules are adsorbed onto the non-protected part of embedded particles. Extraction of embedded particles is done by turning the films upside-down and adding a solvent (sodium hydroxide of higher $\mathrm{pH}$ for hyaluronic acid/poly-L-lysine films). The solvent loosens the interaction between the films and capsules/particles, thus allowing the latter to detach and be collected.

Controlling protrusion of microcapsules into the soft polymeric films and thus controlling the degree of modification or patchiness [41] can be achieved by making the films harder or denser [34], see Figure 2.
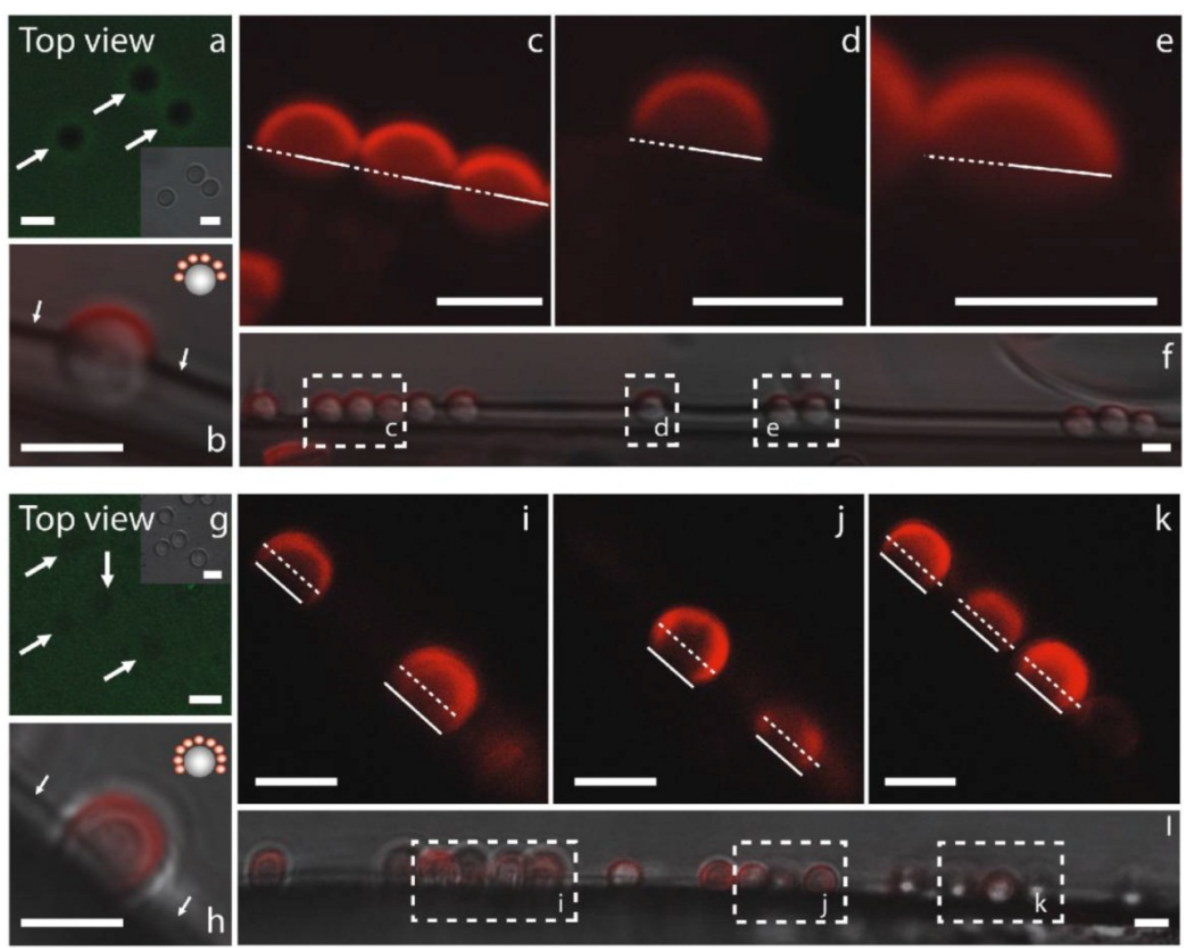

Figure 2. Fluorescence CLSM images showing an overview of a-f) half covered (4.8 $\mu \mathrm{m} \mathrm{SiO}$ )@QDs (quantum dots) and g-l) 2/3 covered $\left(\mathrm{SiO}_{2}\right) @ Q D s$. Dotted lines represent the centre line, while bold lines show the termination of particle modification. Images a) and g) show top view, while c-f) and i-l) depict side view of protruding $\mathrm{SiO}_{2}$ particles into FITC labeled (HA/PLL) 12 films with AuNP concentration of a-f) $0 \mu \mathrm{l}$ and g-l) $300 \mu \mathrm{L}$. Arrows in a) and g) point to location of particles; insets in a) and g) show magnification of the transmission images of protruding $\left(\mathrm{SiO}_{2}\right)$ particles into $(\mathrm{HA} / \mathrm{PLL})_{12}$ films. Arrows in $\left.\mathrm{b}\right)$ and $\mathrm{h}$ ) indicate the (HA/PLL) 12 film height. Scale bars correspond to 5 $\mu \mathrm{m}$. [34] Reproduced by permission of Wiley-VHC. 
This modification can be done by chemical cross-linking or by adding metal nanoparticles onto the surface of the films. While particles can still be embedded into the denser films, they penetrate over a smaller distance [34]. Thus, the masked part of the particles or the degree of patchiness can be controlled through the stiffness of films. This property has been measured by the AFM colloidal probe technique [42, 43]. Peculiarly, such a modification also promotes growth of cells (which grow more effectively on harder substrates) on these soft PLL/HA films as it was recently shown by Schmidt [44]. Anisotropic morphology of particles can be also used for direction-specific release of drug molecules. In such an approach if only a specific part of the microcapsules is functionalized by nanoparticles for triggered release, then release will take place only at the site of nanoparticles. A proof-of-principle of such an approach has been recently demonstrated for giant microgel templated beads [45].

\section{Enzymatic and ionic sensing}

The next step following assembly of the multicompartment capsules is the incorporation of sensing modalities for diagnosis. One can discriminate between two major approaches. The first is encapsulation of larger biomolecules which would serve as sensors for ions. Ions are very small entities which easily penetrate the polyelectrolyte polymeric network. It is this principle that was used for fabrication of polyelectrolyte multilayer capsules: ions penetrating inside the polymeric shell dissolve the template upon which microcapsules are built. Ions are therefore constantly present at equilibrium inside and outside capsules. Monitoring ions allows for instance to assess local $\mathrm{pH}$, which is a very important physiological parameter. For monitoring ions, larger (typically above $1 \mathrm{kD}$ ) fluorescent sensor molecules are encapsulated into the interior of sensing subcompartments. These molecules change their fluorescence characteristics (intensity and/or spectrum) which can be easily observed using fluorescence microscopy [27].

An alternative route for sensing is that based on the detection of products of enzyme-catalyzed reactions. This approach has some similarities to that for the detection of ions: larger sensor molecules are encapsulated inside some subcompartments of multicompartment theranostic capsules. The polymeric mesh allows not only ions, but also small molecules to penetrate through the polymeric network. The larger enzymatic signal molecules change their (often fluorescent) properties which allows for detection of products of enzyme catalyzed reactions [46].

\section{Therapeutic treatment and stimuli for inducing release from capsules}

Therapeutic drugs, such as peptides, proteins and biomolecules are encapsulated into capsules with a polyelectrolyte polymeric mesh. This polymeric mesh permits ions and small molecules to go through, but protects encapsulated drugs which are to be used for treatment. These therapeutic molecules will need to be released $[47,48]$ if the diagnostic signal necessitates so.

Release of molecules can be performed using different physical stimuli, including electromagnetic fields, ultrasound, mechanical force and temperature, Figure 3. Some stimuli possess certain limitations. For example, temperature (global temperature in solution, cell culture or in the body) cannot be substantially changed, thus limiting its application range. Mechanical force is applicable only at specific conditions permitting exerting the force. On the other hand, exerting mechanical forces can be used for measuring the stiffness [49] of capsules and the threshold forces [20] necessary for release. This approach is ideally suited for bench tests of capsules, but it is difficult to exert pressure on capsules in-vivo.

Ultrasound [50] is an interesting physical stimulus for release and some initial work using ultrasound has been conducted to initiate enzyme-catalyzed reactions. Enzyme and substrate were assembled into the same multicompartment microcapsule: the enzyme was held in the interior compartment, while the substrate was incorporated into liposomes attached to the surface of the pericentric multicompartment microcapsules [51]. Upon application of ultrasound, the substrate leaked from the liposomes, penetrated into the interior of the inner particle, initiating the enzyme-catalyzed reaction, Figure 4. An alternative approach is using microcapsules with freely-floating liposomes [52] or multicompartment particles [9], Figure 5. As an example, this approach can be used to fight cancer. An interesting feature of this approach is that both the enzyme and substrate are encapsulated in the same carriers. Calcium carbonate (in the vaterite form) particles used to demonstrate the feasibility of such an approach are biocompatible, porous, and easy to make. Most importantly, it was observed that enzymes immobilized in the pores of particles, were still fully active [51]. Although some progress on reducing the intensity of ultrasound has been recently achieved, a significant hurdle here is to achieve ultrasound mediated release at a power density that is medically acceptable. 


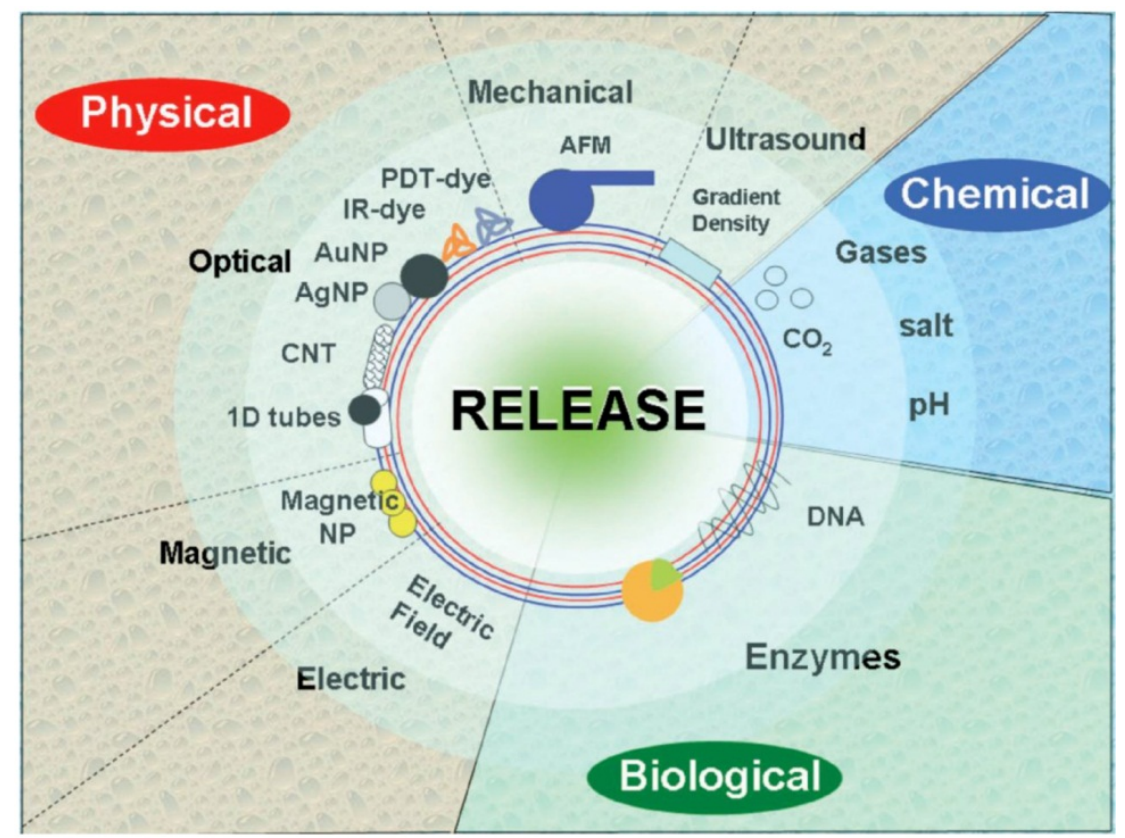

Figure 3. Schematics showing various methods of release. The three main categories: physical, biological and chemical, are further sub-divided and are shown with specific methods of release. [18] Reproduced by permission of The Royal Society of Chemistry.
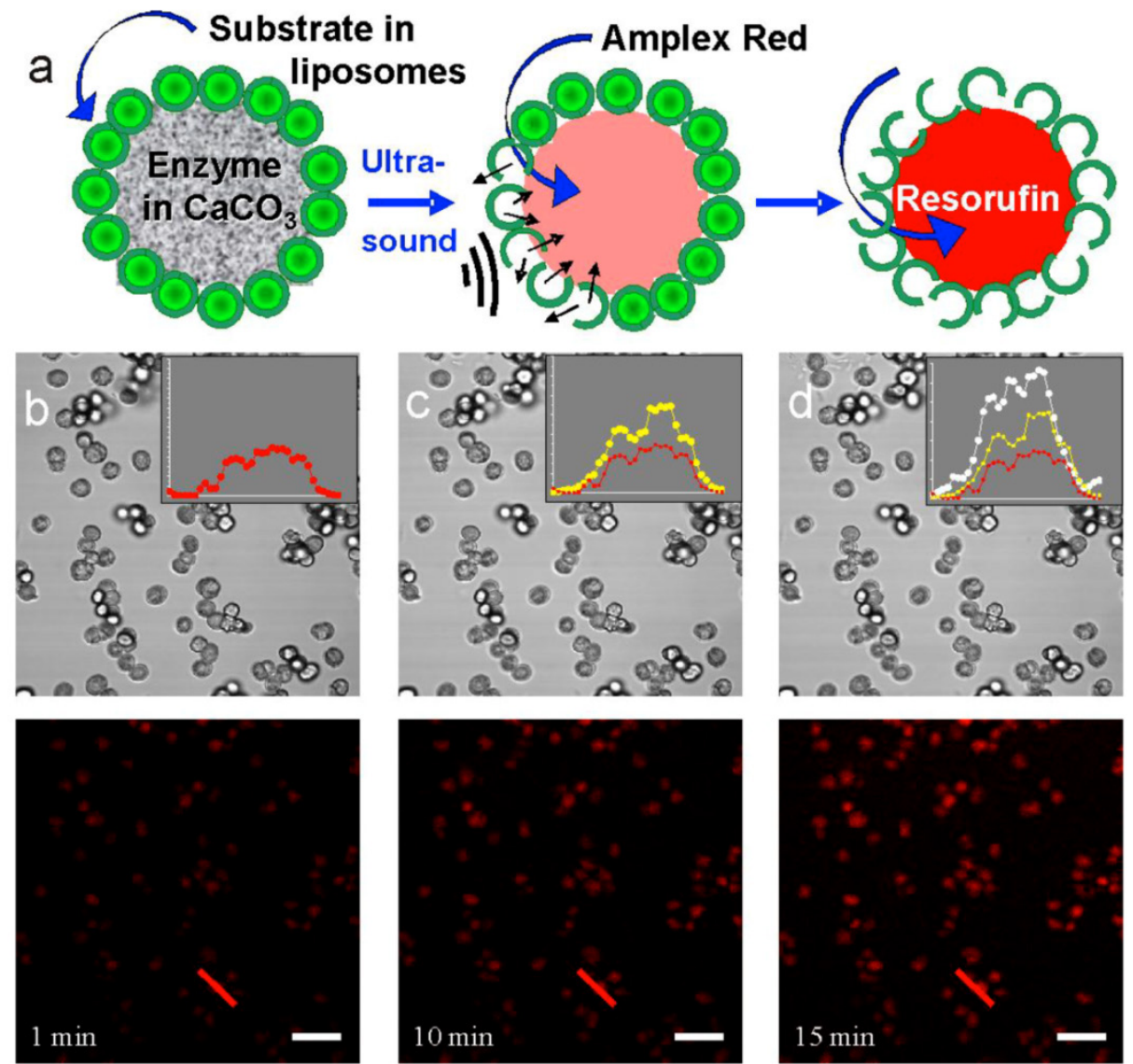

Figure 4. a) Evolution of the enzymatic reaction inside porous microparticles. b-d) Confocal transmission (middle row) and fluorescence (bottom row) images of microparticles after I $\mathrm{min}$ (b), $10 \mathrm{~min}$ (c), and $15 \mathrm{~min}$ (d) of ultrasonication and addition of $\mathrm{H}_{2} \mathrm{O}_{2}$. The insets show fluorescence profiles drawn across the same microcontainer (the red lines in the bottom row). The red profiles in the insets corresponds to emission I min after sonication, the yellow and white profiles to emission 10 and I 5 min after sonication, respectively. [51] Reproduced by permission of Wiley-VHC. 


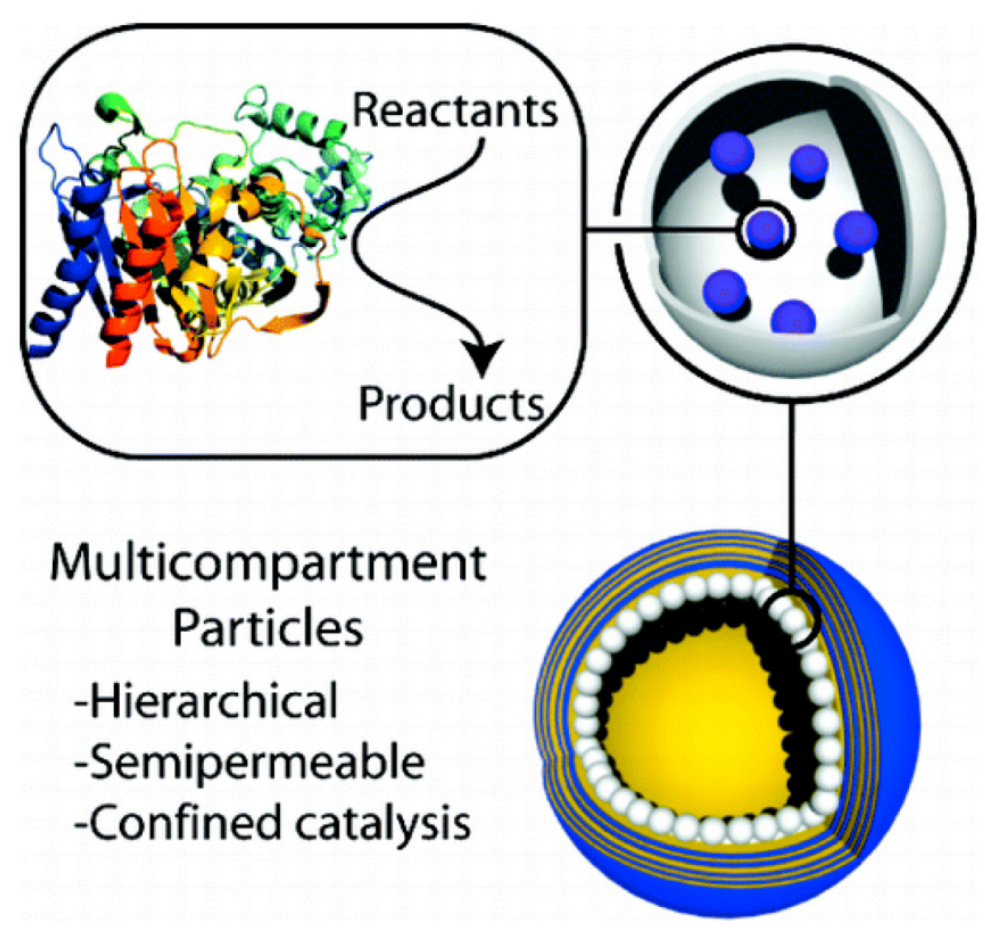

Figure 5. Hierarchy of multicompartment particles for conversion of reactants to products. Reproduced by permission from [9]. Copyright (20II) American Chemical Society.

Electromagnetic energy, such as laser light, can be used as a trigger signal as well. The wavelength used is an important parameter since absorption of laser light by biomolecules is wavelength dependent. UV wavelengths are not useful since penetration in tissue is too limited due to scattering and absorption by biomolecules. Visible light typically cannot be used either since it is already needed for exciting the fluorescent moieties used for sensing and diagnosis. Laser light in the near-infrared range (the so-called biologically "friendly" window [53]) can pass relatively well through water (or a biological medium) so that a reasonable penetration depth in tissue can be obtained. This allows noble metal nanoparticles, such as gold nanorods [54], gold nanoparticles [55] or even carbon nanotubes [56], to be used for exerting a therapeutic action as they can have a strong absorption in the near-infrared range due to the surface plasmon resonance effect. More information on laser-induced release from capsules can be found in the next section.

\section{Laser-induced remotely activated release of encapsulated materials}

Light energy can be converted into heat $[57,58]$ by nanoparticles $[59,60]$. The amount of heat generated can be carefully controlled by tuning the laser intensity, illumination time and the surrounding environment [60]. This effect has been used to induce release from microcapsules [57, 61-63], Figure 6. For example, when irradiated by a continuous wave laser, the nanoparticles will act as local heaters, causing a heat gradient in the surrounding tissue. Here it is important to distinguish between a "global" versus "local" temperature rise. As it was mentioned above it is undesirable to raise the global temperature of biological matter. However, under appropriate illumination conditions, heating can be locally induced without affecting the cell or tissue. It is this localized temperature rise that has been used for inducing transient permeability [64] of the polymeric shell of capsules, thus achieving controlled release of the pharmaceutical cargo. Increasing the concentration of nanoparticles or augmenting the laser power density can lead to increased heat accumulation and therefore to explosive release or activation of microcapsules. It can also be used for hypothermia wherein extensive accumulation of heat is desirable for the killing of cancer cells [65].

Localized, mild temperature rise can be used to obtain a non-destructive permeability change of the capsule walls [64]. An advanced feature here is the possibility of a transient permeability change when the polyelectrolytes of the capsule walls are in the so-called "glassy" state. Localized heating slightly exceeding the "glass" transition temperature renders the polymeric complex flexible. The osmotic pressure of encapsulated materials is enough to push some 
molecules through the polymeric shell. Upon turning off the laser beam, the polymeric complex goes back into the "glassy" state sealing the polymeric shell anew. Thus, the permeability of microcapsules can be regulated transiently upon switching laser: on- and off.

Using laser induced permeability control, release of encapsulated materials has been accomplished inside living cells by microcapsules [53] and directly from particles [55]. Using intracellular release inside living cells, the immune response has been investigated wherein peptides released from the interior of microcapsules were found to form the complex with MHC Class I polymers eliciting movement to the cell surface and the surface presentation [66]. This process is one of the key principles of immune system defense. Cell viability following capsule uptake and triggered release has been verified either by observing cell division or by specific essays, thus proving the feasibility and potential of such an approach for future in vivo use. It can be noted that either stabilized nanoparticles [67] or direct synthesis [68] of nanoparticles on the surfaces can serve to achieve desirable distribution of nanoparticles.

Vapor nanobubble induced by laser nanoparticle interaction in water and biological media have recently proven to offer exciting possibilities, such as the highly specific intracellular delivery [69] and processing [70] of therapeutics. Vapor nanobubbles can be induced by pulsed [71] or continuous wave [72] laser irradiation: the former case is certainly a very promising approach for drug delivery, hypothermia treatment or material modification. Short laser pulses possess specific advantages [73]: high laser peak power and better dissipation of heat. Generating bubbles by continuous wave laser irradiation has been shown applicable for affective soft, polymeric films [72]. A short pulse laser (typically in the order of nanoseconds [74] or less) illuminating metal nanoparticles such as gold nanoparticles leads to extreme localized heating of these nanoparticles due to the excitation of surface plasmon resonance. As a consequence, the spinodal temperature of the surrounding medium, such as water, can be easily surpassed and causes the nucleation of vapor at the nanoparticle surface and the formation of transient nanobubbles. When the thermal energy in consumed, the vapor nanobubble will collapse, causing local pressure waves that can damage nearby tissue. The size of the vapour nanobubbles can be precisely controlled through the laser pulse fluence. It was shown that vapor nanobubbles can aid in drug delivery, cancer treatment and gene therapy.

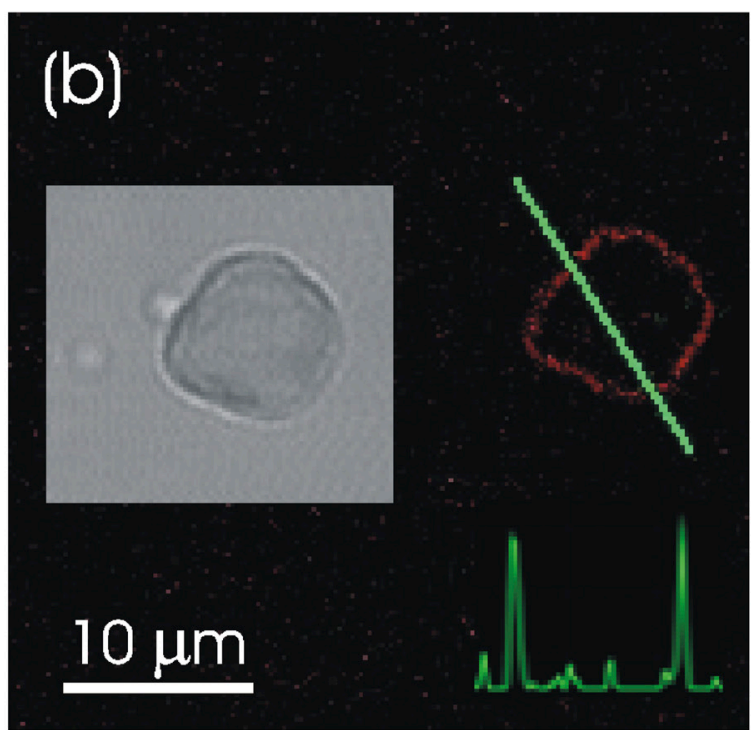

Figure 6. Confocal microscope images demonstrating remote release of encapsulated rhodamine-labeled PSS polymers from a polyelectrolyte multilayer capsule containing gold sulfide core/gold shell nanoparticles in its walls. Fluorescence intensity profiles along the line through the capsule show that it is filled with fluorescent polymers before (a) and empty after (b) laser illumination. After the release of encapsulated polymers, the leftover fluorescent intensity is observed only in the walls of the capsule, (b). Insets show black and white transmission microscope images of the same capsule. Incident intensity of laser diode operating at $830 \mathrm{~nm}$ was set at $50 \mathrm{~mW}$. Reproduced with permission from [57]. Copyright (2005) American Chemical Society. 


\section{Bright perspectives for multicompart- ment capsules for theranostics}

It is envisioned that theranostics will take a unique place in the nanomedicine field in the near future. Precise diagnostics and unique custom initiated, on-demand treatments are the hallmarks of personalized medicine so vigorously being developed now. The use of multicompartment capsules would furthermore allow driving the development of theranostics to another level, Figure 7. A lot of effort is being put in exploring the concept of multimodality, where either different imaging or different therapeutic modalities can be exploited in a combined manner so that any intrinsic shortcomings of a single modality can be compensated for by the strengths of another. Using multicompartment capsules would allow the same entity to be bestowed with several types of imaging contrast agents together with several types of drugs. The different trigger systems also allow the incorporation of various types of therapeutics which can then be selectively released, depending on the opportune time and conditions. As an example, the multicompartment capsules could simultaneously or in a specific order release several anti-HIV drugs to form a more potent cocktail than a single entity. Also, when the same drug would be incorporated in different shells, the use of various stimuli at different time points would allow a repeated release of high levels of the drug at the target site, which could offer many advantages over a single bolus injection or repeated injections of the drug. Further research and developmental work, including in vivo studies, will surely contribute to its wide applicability in biomedicine.

Further assembly of microcompartment capsules can be based not only on synthetic carriers, but also based on red blood cells [75, 76] from which release has also been recently demonstrated [77].

\section{Conclusions}

Theranostics is an emerging area which combines diagnostics and therapy. It will allow physicians not only to detect diseases, but will also permit them to adequately administer medicines, thus paving the way to personalized medicine. Multicompartment microcapsules are ideal candidates for application in theranostics owing to the multifunctionality by the constituting subcompartments. Indeed, therapeutic compounds can be incorporated inside some subcompartments, while other subcompartments can be filled with sensing agents, which are to be used for sensing and diagnosis. A particular attractive property of such elaborate drug delivery carriers is that they allow for diagnostics and subsequent treatment by implementing release of drugs at a desired site and rate. Laser light, in combination with noble metal nanoparticles, has proven to be a particularly attractive for achieving localized triggered release. Further developments of light sensitive drug carriers, and capsules in general [78], are expected to impact the area of theranostics and nanomedicine in the future.

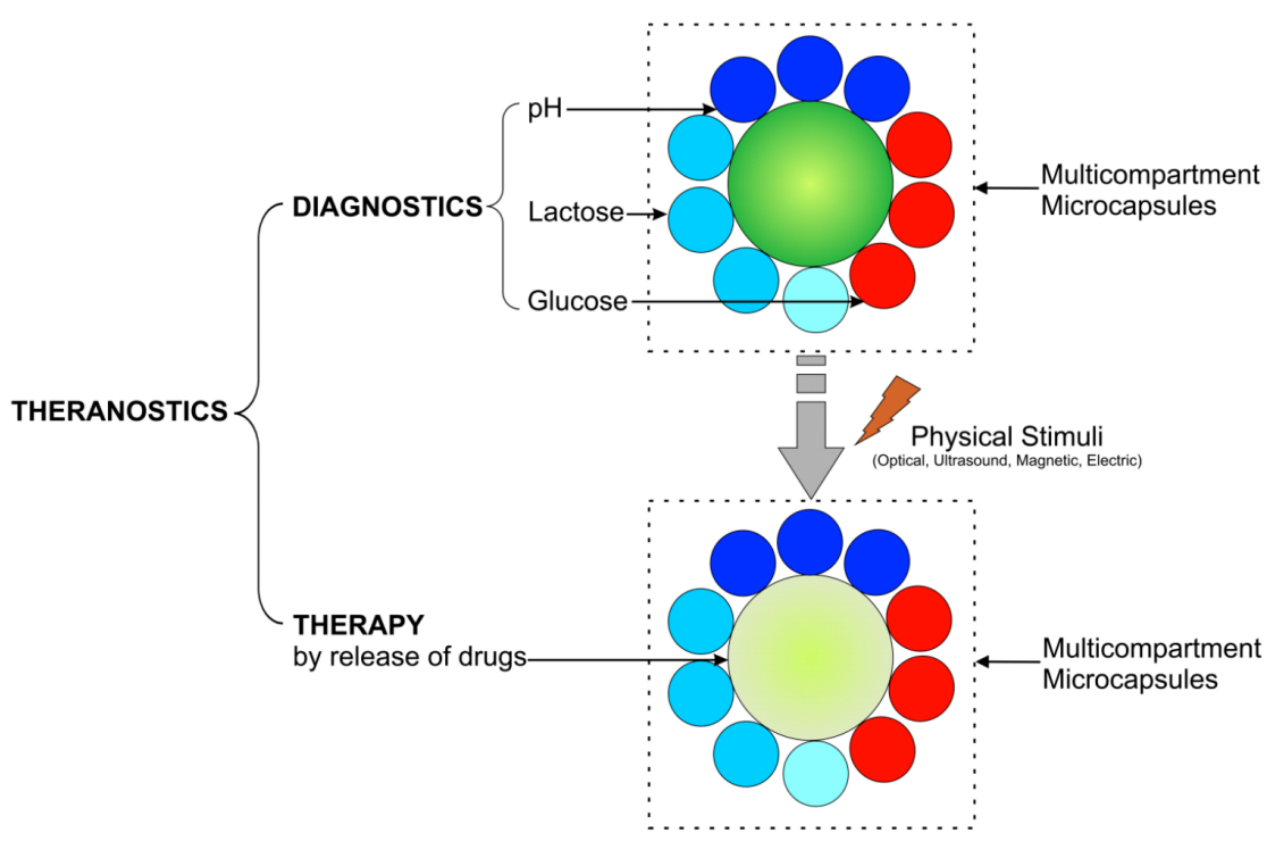

Figure 7. Schematics of implementation of theranostic multicompartment capsules: diagnostics is achieved by monitoring $\mathrm{pH}$, glucose, lactose, etc., while therapy is attained by release of encapsulated drugs/medicine. 


\section{Acknowledgments}

We thank FWO (Fonds Wetenschappelijk Onderzoek), the Belgian Research Council, for support of this work.

\section{Competing Interests}

The authors have declared that no competing interest exists.

\section{References}

[1] Delcea M, Yashchenok A, Videnova K, Kreft O, Mohwald H, Skirtach AG. Multicompartmental micro- and nanocapsules: hierarchy and applications in biosciences. Macromol Biosci. 2010; 10: 465-474.

[2] Vergaro V, Baldassarre F, De Santis F, Ciccarella G, Giannelli G, Leporatti S. TGF-beta inihibitor-loaded polyelectrolyte multilayers capsules for sustained targeting of hepatocarcinoma cells. Curr Pharm Design. 2012; 18: 4155-4164.

[3] Lukianova-Hleb EY, Oginsky AO, Samaniego AP, Shenefelt DL, Wagner DS, Hafner JH, Farach-Carson MC, Lapotko DO. Tunable plasmonic nanoprobes for theranostics of prostate cancer. Theranostics. 2011; 1: 3-17.

[4] Gao L, Fei JB, Zhao J, Li H, Cui Y, Li JB. Hypocrellin-loaded gold nanocages with high two-photon efficiency for photothermal/photodynamic cancer therapy in vitro. ACS Nano. 2012; 6: 8030-8040.

[5] Tong R, Kohane DS. Shedding light on nanomedicine. Nanomed Nanobiotechnol. 2012; 4: 638-662.

[6] Amstad E, Reimhult E. Nanoparticle actuated hollow drug delivery vehicles. Nanomedicine. 2012; 7 : 145-164.

[7] Delcea M, Mohwald H, Skirtach AG. Stimuli-responsive LbL capsules and nanoshells for drug delivery. Adv Drug Deliv Rev. 2011; 63: 730-747.

[8] Marguet M., Bonduelle C. Lecommandoux S. Multicompartmentalized polymeric systems: towards biomimetic cellular structure and function. Chem Soc Rev. 2013; 42: 512-529.

[9] Chandrawati $R$, van Koeverden MP, Lomas H, Caruso F. Multicompartment particle assemblies for bioinspired encapsulated reactions. J Phys Chem Lett. 2011; 2: 2639-2649.

[10] Murthy VS, Kadali SB, Wong MS. Polyamine-guided synthesis of anisotropic, multicompartment microparticles. ACS Appl Mater Interfaces. 2009; 1: 590-596.

[11] Chandrawati R, Caruso F. Biomimetic liposome- and polymersome-based multicompartmentalized assemblies. Langmuir. 2012; 28: 13798-13807.

[12] Stadler B, Chandrawati R, Price AD, Chong SF, Breheney K, Postma A, Connal LA, Zelikin AN, Caruso F. A microreactor with thousands of subcompartments: enzyme-loaded liposomes within polymer capsules. Angew Chem Int Edit. 2009; 48: 4359-4362.

[13] Boyer C, Zasadzinski JA. Multiple lipid compartments slow vesicle contents release in lipases and serum. ACS Nano. 2007; 1: 176-182.

[14] de Hoog HPM, Nallani M, Tomczak N. Self-assembled architectures with multiple aqueous compartments. Soft Matter. 2012; 8: 4552-4561.

[15] Huang X, Voit B. Progress on multi-compartment polymeric capsules. Polym chem. 2013; 4: 435-443.

[16] Shchepelina O, Lisunova MO, Drachuk I, Tsukruk VV. Morphology and properties of microcapsules with different core releases. Chem Mater. 2012; 24: 1245-1254.

[17] Berg MC, Zhai L, Cohen RE, Rubner MF. Controlled drug release from porous polyelectrolyte multilayers. Biomacromol. 2006; 7: 357-364.

[18] Skirtach AG, Yashchenok AM, Mohwald H. Encapsulation, release and applications of LbL polyelectrolyte multilayer capsules. Chem Commun. 2011; 47: 12736-12746.

[19] Ye CH, Drachuk I, Calabrese R, Dai HQ, Kaplan DL, Tsukruk VV. Permeability and micromechanical properties of silk ionomer microcapsules. Langmuir. 2012; 28: 12235-12244.

[20] Lee WL, Loo SCJ. Revolutionizing drug delivery through biodegradable multilayered particles. J Drug Target. 2012; 20: 633-647.

[21] Bocharova V, Zavalov O, MacVittie K, Arugula MA, Guz NV, Dokukin ME, Halamek J, Sokolov I, Privman V, Katz E. A biochemical logic approach to biomarker-activated drug release. J Mater Chem. 2012; 22: 19709-19717.
[22] Marchenko I, Yashchenok A, Borodina T, Bukreeva T, Konrad M, Mohwald H, Skirtach A. Controlled enzyme-catalyzed degradation of polymeric capsules templated on $\mathrm{CaCO}_{3}$ : Influence of the number of $\mathrm{LbL}$ layers, conditions of degradation, and disassembly of multicompartments. J Control Release. 2012; 162: 599-605.

[23] De Geest BG, Vandenbroucke RE, Guenther AM, Sukhorukov GB, Hennink WE, Sanders NN, Demeester J, De Smedt SC. Intracellularly degradable polyelectrolyte microcapsules. Adv Mater. 2006; 18: 1005-1009.

[24] Baumler H, Georgieva R. Coupled enzyme reactions in multicompartment microparticles. Biomacromol. 2010; 11: 1480-1487.

[25] Drachuk I, Shchepelina O, Lisunova M, Harbaugh S, Kelley-Loughnane $\mathrm{N}$, Stone M, Tsukruk VV. pH-responsive layer-by-layer nanoshells for direct regulation of cell activity. ACS Nano. 2012; 6: 4266-4278.

[26] Yashchenok AM, Borisova D, Parakhonskiy BV, Masic A, Pinchasik BE, Mohwald H, Skirtach AG. Nanoplasmonic smooth silica versus porous calcium carbonate bead biosensors for detection of biomarkers. Ann Phys. 2012; 524: 723-732.

[27] Kreft O, Javier AM, Sukhorukov GB, Parak WJ. Polymer microcapsules as mobile local pH-sensors. J Mater Chem. 2007; 17: 4471-4476.

[28] Kida T, Mouri M, Kondo K, Akashi M. Controlled release using a polymer stereocomplex capsule through the selective extraction and incorporation of one capsule shell component. Langmuir. 2012; 28: 15378-15384.

[29] Kreft O, Prevot M, Mohwald H, Sukhorukov GB. Shell-in-shell microcapsules: A novel tool for integrated, spatially confined enzymatic reactions. Angew Chem Int Edit. 2007; 46: 5605-5608.

[30] Delcea M, Madaboosi N, Yashchenok AM, Subedi P, Volodkin DV, De Geest BG, Mohwald H, Skirtach AG. Anisotropic multicompartment micro- and nano-capsules produced via embedding into biocompatible PLL/HA films. Chem Commun. 2011; 47: 2098-2100.

[31] Chow AHL, Tong HHY, Chattopadhyay P, Shekunov BY. Particle engineering for pulmonary drug delivery. Pharm Res. 2007; 24: 411-437.

[32] Kozlovskaya V, Wang Y, Higgins W, Chen J, Chen Y, Kharlampieva E. $\mathrm{pH}$-triggered shape response of cubical ultrathin hydrogel capsules. Soft Matter. 2012; 8: 9828-9839.

[33] Jiang S, Chen Q, Tripathy M, Luijten E, Schweizer KS, Granick S. Janus particle synthesis and assembly. Adv Mater. 2010; 22: 1060-1071.

[34] Kohler D, Madaboosi N, Delcea M, Schmidt S, De Geest BG, Volodkin DV, Mohwald H, Skirtach AG. Patchiness of embedded particles and film stiffness control through concentration of gold nanoparticles. Adv Mater. 2012; 24: 1095-1100.

[35] Staff RH, Gallei M, Mazurowski M, Rehahn M, Berger R, Landfester K, Crespy D. Patchy nanocapsules of poly(vinylferrocene)-based block copolymers for redox-responsive release. ACS Nano. 2012; 6: 9042-9049.

[36] Nisisako T, Torii T, Takahashi T, Takizawa Y. Synthesis of monodisperse bicolored janus particles with electrical anisotropy using a microfluidic co-flow system. Adv Mater. 2006; 18: 1152-1156.

[37] Cui JQ, Kretzschmar I. Surface-anisotropic polystyrene spheres by electroless deposition. Langmuir, 2006;22: 8281-8284.

[38] Cayre O, Paunov VN, Velev OD. Fabrication of dipolar colloid particles by microcontact printing. Chem Commun. 2003;:2296-2297. doi: 10.1039/B307296G.

[39] Berger S, Synytska A, Ionov L, Eichhorn KJ, Stamm M. Stimuli-responsive bicomponent polymer Janus particles by "grafting from" /"grafting to" approaches. Macromolecules. 2008; 41: 9669-9676.

[40] Anderson KD, Luo MD, Jakubiak R, Naik RR, Bunning TJ, Tsukruk VV. Robust plasma polymerized-titania/silica Janus microparticles. Chem Mater. 2010; 22: 3259-3264.

[41] Ling XY, Phang IY, Acikgoz C, Yilmaz MD, Hempenius MA, Vancso GJ, Huskens J. Janus particles with controllable patchiness and their chemical functionalization and supramolecular assembly. Angew Chem Int Edit. 2009; 48: 7677-7682.

[42] Fernandes PAL, Delcea M, Skirtach AG, Mohwald H, Fery A. Quantification of release from microcapsules upon mechanical deformation with AFM. Soft Matter. 2010; 6: 1879-1883.

[43] Fery A, Weinkamer R. Mechanical properties of micro- and nanocapsules: Single-capsule measurements. Polymer. 2007; 48: 7221-7235.

[44] Schmidt S, Madaboosi N, Uhlig K, Kohler D, Skirtach A, Duschl C, Mohwald H, Volodkin DV. Control of cell adhesion by mechanical reinforcement of soft polyelectrolyte films with nanoparticles. Langmuir. 2012; 28: 7249-7257.

[45] Bedard MF, De Geest BG, Moehwald H, Sukhorukov GB, Skirtach AG. Direction specific release from giant microgel-templated polyelectrolyte microcontainers. Soft Matter. 2009; 5: 3927-3931. 
[46] Kazakova LI, Shabarchina LI, Anastasova S, Pavlov AM, Vadgama P, Skirtach AG, Sukhorukov GB. Chemosensors and biosensors based on polyelectrolyte microcapsules containing fluorescent dyes and enzymes. Anal Bioanal Chem. 2013; doi: 10.1007/s00216-00012-06381-00210.

[47] Leung SJ, Romanowski M. Light-activated content release from liposomes. Theranostics. 2012; 2: 1020-1036.

[48] Reibetanz U, Schonberg M, Rathmann S, Strehlow V, Gose M, Lessig J. Inhibition of human neutrophil elastase by alpha(1)-antitrypsin functionalized colloidal microcarriers. ACS Nano. 2012; 6: 6325-6336.

[49] Bedard MF, Munoz-Javier A, Mueller R, del Pino P, Fery A, Parak WJ, Skirtach AG, Sukhorukov GB. On the mechanical stability of polymeric microcontainers functionalized with nanoparticles. Soft Matter. 2009; 5: 148-155.

[50] Anandhakumar S, Mahalakshmi V, Raichur AM. Silver nanoparticles modified nanocapsules for ultrasonically activated drug delivery. Mater Sci Eng C-Mater Biol Appl. 2012; 32: 2349-2355.

[51] Yashchenok AM, Delcea M, Videnova K, Jares-Erijman EA, Jovin TM, Konrad M, Mohwald H, Skirtach AG. Enzyme reaction in the pores of $\mathrm{CaCO}_{3}$ particles upon ultrasound disruption of attached substrate-filled liposomes. Angew Chem Int Edit. 2010; 49: 8116-8120.

[52] Hosta-Rigau L, Chung SF, Postma A, Chandrawati R, Stadler B, Caruso F. Capsosomes with "free-floating" liposomal subcompartments. Adv Mater. 2011; 23: 4082-4087.

[53] Skirtach AG, Munoz Javier A, Kreft O, Kohler K, Piera Alberola A, Mohwald H, Parak WJ, Sukhorukov GB. Laser-induced release of encapsulated materials inside living cells. Angew Chem Int Edit. 2006; 45: 4612-4617.

[54] Huang JY, Jackson KS, Murphy CJ. Polyelectrolyte wrapping layers control rates of photothermal molecular release from gold nanorods. Nano Lett. 2012; 12: 2982-2987.

[55] Huschka R, Neumann O, Barhoumi A, Halas NJ. Visualizing light-triggered release of molecules inside living cells. Nano Lett. 2010; 10: 4117-4122.

[56] Yashchenok AM, Bratashov DN, Gorin DA, Lomova MV, Pavlov AM, Sapelkin AV, Shim BS, Khomutov GB, Kotov NA, Sukhorukov GB, Möhwald H, Skirtach AG. Carbon nanotubes on polymeric microcapsules: free-standing structures and point-wise laser openings. Adv Funct Mater. 2010; 20: 3136-3142

[57] Skirtach AG, Dejugnat C, Braun D, Susha AS, Rogach AL, Parak WJ, Mohwald H, Sukhorukov GB. The role of metal nanoparticles in remote release of encapsulated materials. Nano Lett. 2005; 5: 1371-1377.

[58] Alessandri I, Depero LE. Using plasmonic heating of gold nanoparticles to generate local SER(R)S-active $\mathrm{TiO}(2)$ spots. Chem Commun. 2009;: 2359-2361. doi: 10.1039/B900647H

[59] Urban AS, Fedoruk M, Horton MR, Radler J, Stefani FD, Feldmann J. Controlled nanometric phase transitions of phospholipid membranes by plasmonic heating of single gold nanoparticles. Nano Lett. 2009; 9: 2903-2908.

[60] Skorb EV, Skirtach AG, Sviridov DV, Shchukin DG, Mohwald H. Laser-controllable coatings for corrosion protection. ACS Nano. 2009; 3: 1753-1760.

[61] Skirtach AG, Antipov AA, Shchukin DG, Sukhorukov GB. Remote activation of capsules containing Ag nanoparticles and IR dye by laser light. Langmuir. 2004; 20: 6988-6992.

[62] Radt B, Smith TA, Caruso F. Optically addressable nanostructured capsules. Adv Mater. 2004; 16: 2184-2189.

[63] Angelatos AS, Radt B, Caruso F. Light-responsive polyelectrolyte / gold nanoparticle microcapsules. J Phys Chem B. 2005; 109: 3071-3076.

[64] Skirtach AG, Karageorgiev P, Bedard MF, Sukhorukov GB, Mohwald H. Reversibly permeable nanomembranes of polymeric microcapsules. J Am Chem Soc. 2008; 130: 11572-11573.

[65] Javier AM, del Pino P, Bedard MF, Ho D, Skirtach AG, Sukhorukov GB, Plank C, Parak WJ. Photoactivated release of cargo from the cavity of polyelectrolyte capsules to the cytosol of cells. Langmuir. 2008; 24: 12517-12520.

[66] Palankar R, Skirtach AG, Kreft O, Bedard M, Garstka M, Gould K, Mohwald H, Sukhorukov GB, Winterhalter M, Springer S. Controlled intracellular release of peptides from microcapsules enhances antigen presentation on MHC class I molecules. Small. 2009; 5: 2168-2176.

[67] Radziuk D, Skirtach A, Sukhorukov G, Shchukin D, Mohwald H. Stabilization of silver nanoparticles by polyelectrolytes and poly(ethylene glycol. Macromol Rapid Commun. 2007; 28: 848-855.

[68] Parakhonskiy BV, Bedard MF, Bukreeva TV, Sukhorukov GB, Mohwald H, Skirtach AG. Nanoparticles on polyelectrolytes at low concentration: controlling concentration and size. J Phys Chem C. 2010; 114: 1996-2002.
[69] Lukianova-Hleb EY, Samaniego AP, Wen JG, Metelitsa LS, Chang CC, Lapotko DO. Selective gene transfection of individual cells in vitro with plasmonic nanobubbles. J Control Release. 2011; 152: 286-293.

[70] Lukianova-Hleb EY, Mutonga MBG, Lapotko DO. Cell-specific multifunctional processing of heterogeneous cell systems in a single laser pulse treatment. ACS Nano. 2012; 6: 10973-10981.

[71] Lukianova-Hleb EY, Hanna EY, Hafner JH, Lapotko DO. Tunable plasmonic nanobubbles for cell theranostics. Nanotechnology. 2010; 21: 85102.

[72] Volodkin DV, Delcea M, Mohwald H, Skirtach AG. Remote near-IR light activation of a hyaluronic acid/poly(L-lysine) multilayered film and film-entrapped microcapsules. ACS Appl Mater Interfaces 2009; 1: 1705-1710.

[73] Baffou G, Rigneault H. Femtosecond-pulsed optical heating of gold nanoparticles. Phys Rev B. 2011; 84: 035415.

[74] Siems A, Weber SAL, Boneberg J, Plech A. Thermodynamics of nanosecond nanobubble formation at laser-excited metal nanoparticles. New J Phys. 2011; 13: 043018.

[75] Muzykantov VR. Drug delivery by red blood cells: vascular carriers designed by mother nature. Expert Opin Drug Deliv. 2010; 7: 403-427.

[76] Kolesnikova TA, Skirtach AG, Mohwald H. Red blood cells and polyelectrolyte multilayer capsules: natural carriers versus polymer-based drug delivery vehicles. Expert Opin Drug Deliv. 2013; 10: 47-58.

[77] Delcea M, Sternberg N, Yashchenok AM, Georgieva R, Baumler H, Mohwald H, Skirtach AG. Nanoplasmonics for dual-molecule release through nanopores in the membrane of red blood cells. ACS Nano. 2012; 6: 4169-4180.

[78] Tong WJ, Song XX, Gao CY. Layer-by-layer assembly of microcapsules and their biomedical applications. Chem Soc Rev. 2012; 41: 6103-6124. 